\title{
Cambios alimenticios en mujeres morelenses migrantes a Estados Unidos
}

\author{
Luz Arenas-Monreal, D en Antropol,(') Myriam Ruiz-Rodríguez, D en Sist de Salud,(2) \\ Pastor Bonilla-Fernández, M en C,(I) Rosario Valdez Santiago, D en Sist de Salud,(I) \\ Isabel Hernández-Tezoquipa, D en Sociol.t,(I)
}

\section{Arenas-Monreal L, Ruiz-Rodríguez M, Bonilla-Fernández P, Valdez Santiago R, Hernández-Tezoquipa I. Cambios alimenticios en mujeres morelenses migrantes a Estados Unidos. Salud Publica Mex 2013;55:35-42.}

\begin{abstract}
Resumen
Objetivo. Describir la modificación de la alimentación y los procesos involucrados en la incorporación de nuevos alimentos, en mujeres mexicanas con experiencia migratoria hacia Estados Unidos de América. Material y métodos. Estudio cualitativo basado en la teoría fundamentada, en el cual participaron mujeres con experiencia migratoria, residentes de zonas rurales de municipios del Estado de Morelos. Los datos fueron obtenidos a partir de 47 entrevistas en profundidad, realizadas entre febrero de 2005 y julio de 2006. Resultados. La alimentación de las mujeres se modifica a partir de la experiencia migratoria, ya que se incrementa la cantidad de su consumo durante su estancia en Estados Unidos. Continúan cocinando comidas tradicionales mexicanas, pero incorporan alimentos del país de destino, lo cual se ve favorecido por el mayor poder de compra, aspectos laborales, y el acceso tanto a otro tipo de productos alimentarios, como a los diferentes espacios en los que se expende comida. Conclusiones. Es necesario profundizar en la relación de alimentación-migración en el contexto de México-Estados Unidos-México y en su impacto sobre la salud de las mujeres, además de proponer políticas públicas dirigidas a fortalecer sus hábitos saludables.
\end{abstract}

Palabras clave: mujeres; prácticas alimenticias; migración México-Estados Unidos
Arenas-Monreal L, Ruiz-Rodríguez M, Bonilla-Fernández P, Valdez Santiago R, Hernández-Tezoquipa I.

Dietary changes in mexican women

migrating to the U.S.

Salud Publica Mex 2013;55:35-42.

\begin{abstract}
Objective. To describe the dietary changes and processes involved in the incorporation of new foods, in Mexican women with a migratory experience to the U.S. Material and methods. A qualitative study was conducted, based on grounded theory, of women who had had a migratory experience and were residing in rural zones of the state of Morelos, Mexico. The data were obtained from 47 in-depth interviews carried out from February, 2005 to July, 2006. Results. The women's diet is modified by the migratory experience and the amount of consumption increases during their stay in the U.S. They continue cooking traditional Mexican meals but incorporate foods from their country of destination. These changes are favored by the greater purchasing power, work-related aspects and availability of products and spaces where food is sold. Conclusions. We need to go deeper into the relationship between food and migration in the context of Mexico - U.S. - Mexico migration and its impact on women's health. We also need to propose public policies geared towards strengthening healthy habits.
\end{abstract}

Key words: women; food and nutrition practices; MexicanAmerican migration

(I) Centro de Investigaciones en Sistemas de Salud, Instituto Nacional de Salud Pública, México.

(2) Departamento de Salud Pública. Universidad Industrial de Santander. Bucaramanga, Colombia.

Fecha de recibido: 20 de septiembre de 20II • Fecha de aceptado: 7 de septiembre de 2012

Autor de correspondencia: Dra. Luz Arenas-Monreal. Instituto Nacional de Salud Pública. Centro de Investigaciones en Sistemas de Salud.

Av. Universidad 655, col. Santa María Ahuacatitlán, 62100 Cuernavaca, Morelos, México.

Correo electrónico: mareanas@insp.mx 
$E^{n}$ las últimas décadas, el fenómeno migratorio ha cobrado vigencia en el ámbito mundial. Para el año 2005 existían 190633564 migrantes internacionales, de los cuales, $49.6 \%$ eran mujeres. ${ }^{1}$

Todos los continentes se encuentran inmersos en los procesos migratorios, ya sea como expulsores o receptores de población. De los migrantes internacionales, 25\% se encuentra en Asia; $23.3 \%$, en Estados Unidos; 18.75\%, en Europa; 16.8\%, en la antigua Unión Soviética( URSS); 9.3\%, en África; 3.3\%, en América Latina, y 3.45\%, en Oceanía. ${ }^{2}$

Respecto a la migración de mujeres es pertinente ubicarla bajo la perspectiva de género, debido a que los movimientos migratorios están mediados por la construcción cultural diferenciada entre lo femenino y masculino, lo que determina las motivaciones y características de la migración de hombres y mujeres. En el caso de la migración femenina de México a Estados Unidos, en general las mujeres migrantes presentan el "patrón tradicional", es decir, migran por cuestiones familiares, y las redes de apoyo juegan un papel primordial.,

La migración en cualquiera de sus modalidades tiene implicaciones diversas para la salud pública. En los últimos años se han publicado varios estudios que señalan los cambios que se presentan en la alimentación de las personas migrantes de países pobres a países ricos. ${ }^{5-13}$

S. Menell y colaboradores señalan que uno de los resultados de las migraciones humanas ha sido el intercambio de alimentos, y mencionan tres grandes acontecimientos que han contribuido a dispersar diversos productos alimenticios en los distintos continentes: a) la llegada de los europeos al continente americano; b) la revolución industrial, y c) la segunda guerra mundial. ${ }^{14}$ La alimentación es parte de la identidad de los distintos grupos humanos, y varios autores señalan que los migrantes tratan de mantener su dieta y sus formas de cocinar el mayor tiempo posible, a pesar de las presiones de que son objeto en los países a los que migran. ${ }^{15,16} \mathrm{M}$. Calvo analiza la alimentación entre los migrantes en Europa y señala que la inserción económica, la identidad cultural y la familia, así como los elementos estructurales del país al que migran son aspectos fundamentales que influyen en la reorganización de la dieta a la que se encuentran sujetas las poblaciones migrantes. ${ }^{17}$

La teoría de la trasnacionalización, que se refiere al fenómeno migratorio a través de las fronteras, y en el cual la pertenencia étnica juega un papel importante, aporta elementos para el análisis de lo que ocurre con la alimentación y la migración. Dentro de este marco se habla de comunidades trasnacionales, medio a través del cual los migrantes hacen frente a las dificultades de inserción social en las zonas receptoras al vincularse, tanto en el país de origen como en el de destino, con personas de su mismo origen. Estos lazos aportan un sentimiento de identidad y pertenencia comunitaria, en el cual los alimentos se ubican en un lugar central. ${ }^{18,19}$

La situación de la migración interna y de la alimentación presentan características diferentes. En la migración de las zonas rurales a las urbanas en México, los cambios en la alimentación consisten en un consumo de mayor abundancia y variedad, debido a que los migrantes cuentan con mayores recursos económicos y disponibilidad de alimentos; más que una sustitución, lo que se observa es una adición de nuevos productos. ${ }^{20}$ Sin embargo, hay divergencia con este señalamiento, ya que otros autores mencionan que la calidad de la dieta no mejora a pesar de la migración a zonas urbanas. ${ }^{21}$

Revisar lo que acontece en los cambios de las prácticas alimenticias de las mujeres migrantes tiene importancia, no solo por lo que este fenómeno puede representar para la salud de la propia mujer, sino para las familias y grupos sociales en relación con la función de la mujer en el interior de las familias; además, la visualización de este hecho social puede contribuir para que se diseñen iniciativas de promoción de la salud y políticas públicas, dirigidas tanto a fortalecer prácticas de alimentación saludables como a limitar aquellas que son perjudiciales.

El objetivo de este artículo es describir la modificación en la alimentación y los procesos involucrados en la incorporación de nuevos alimentos en mujeres mexicanas con experiencia migratoria hacia Estados Unidos de América.

\section{Material y métodos}

Estudio cualitativo con métodos de la teoría fundamentada. ${ }^{22}$ Los datos se obtuvieron por medio de 47 entrevistas en profundidad, realizadas entre febrero de 2005 y julio de 2006, en localidades rurales de cinco municipios (Miacatlán, Tepalcingo, Tlayacapan, Totolapan y Yautepec) ubicados en el Estado de Morelos. Estas regiones son consideradas de mediana y alta migración hacia Estados Unidos. Los estados a los que emigran son California, Florida, Texas, Utah y Washington.

\section{Procedimiento}

Previo al inicio del estudio, se diseñó una guía de entrevista en profundidad y se estableció contacto con mujeres, grupos comunitarios y autoridades locales de los municipios mencionados. Las participantes fueron contactadas por medio de la técnica de bola de nieve. Las entrevistas se efectuaron por tres integrantes del equipo 
de investigación (IHT, LAM, MRR) en las viviendas de las mujeres o en espacios comunitarios.

\section{Participantes}

Se entrevistaron 47 mujeres con experiencia migratoria hacia Estados Unidos y con una estancia de, por lo menos, dos años de duración. La entrevista se realizó en una sola ocasión y tuvo una duración de 90-120 minutos. El rango de edad de las mujeres entrevistadas fue de 18-86 años. Solamente en tres mujeres no se obtuvieron los datos socio demográficos (cuadro I) .

\section{Consideraciones éticas}

El estudio fue aprobado por las Comisiones de Investigación y Ética del Instituto Nacional de Salud Pública. A cada participante se le dio una carta, solicitando su consentimiento, y en la cual se enunciaban los fines del estudio, la confidencialidad de la información y se

Cuadro I

\section{Características sociodemográficas de mujeres de I 8-86 AÑOS DE EDAD, PARTICIPANTES EN EL ESTUDIO $\mathbf{N}=44$}

\begin{tabular}{|c|c|}
\hline Caracteristicas & $n / \%$ \\
\hline \multicolumn{2}{|l|}{ Estado civil } \\
\hline Con pareja (unión libre o casada) & $32 / 72$ \\
\hline Separada o viuda & $11 / 25$ \\
\hline Soltera & $1 / 3$ \\
\hline Número de hijos reportado por $53 \%$ de participantes & 2 a 4 hijos \\
\hline \multicolumn{2}{|l|}{ Ocupación en México } \\
\hline \multicolumn{2}{|l|}{ Ama de casa, empleada doméstica, } \\
\hline labores agrícolas, comercio informal & 15/34.I \\
\hline Exclusivamente hogar & $18 / 40.9$ \\
\hline \multicolumn{2}{|l|}{ Otras actividades } \\
\hline (negocio propio, empleada en servicios) & 10/22.7 \\
\hline No trabaja (incapacidad por artritis) & $1 / 2.3$ \\
\hline \multicolumn{2}{|l|}{ Actividad laboral en Estados Unidos } \\
\hline Restaurantes y/o venta de alimentos & $|4 / 3| .8$ \\
\hline Fábricas (de costura, pilas, entre otras) & $5 / 11.4$ \\
\hline \multicolumn{2}{|l|}{ Otras: } \\
\hline Agrícolas, trabajo doméstico, hoteles, & \\
\hline cuidadoras de niños y enfermos & $23 / 52.3$ \\
\hline No trabajó & $2 / 4.5$ \\
\hline
\end{tabular}

pedía autorización para audiograbar las entrevistas. A los casetes de las entrevistas se les colocó un registro numérico, sin los nombres de las entrevistadas.

\section{Análisis}

Las entrevistas fueron transcritas en un procesador de textos y codificadas a través del programa de cómputo Ethnograph v.5. Las fases en las que se dividió su análisis fueron las siguientes: 1) Lectura de las entrevistas y elección de códigos; 2) Discusión y acuerdos entre el grupo de investigación acerca de los códigos, 3) Nueva lectura de entrevistas y codificación, 4) Análisis por códigos, diferenciando cada entrevista para encontrar regularidades y diferencias, y 5) Elaboración de tablas de concentración y matrices de datos a partir de la información encontrada en las entrevistas.

El análisis incluyó el proceso de codificación abierta, codificación axial y el desarrollo de una matriz condicional que representaba las condiciones, consecuencias y acciones del fenómeno en estudio. La saturación teórica se alcanzó en el análisis de las entrevistas, a partir del cual se encontró un esquema teórico que permitió identificar la categoría central, como el fenómeno alrededor del cual estaban integradas las otras categorías. La central fue el traslado social de prácticas alimenticias. Para los fines de este estudio, se definió como el proceso dinámico mediante el cual, las mujeres, cuando migran hacia Estados Unidos, llevan una serie de prácticas alimenticias, algunas de las cuales permanecen durante su estancia con una serie de ajustes a la dieta, mediante la incorporación de otros alimentos pertenecientes a la cultura alimenticia de ese país.

En este manuscrito se presentan la categoría central y las vinculadas que dan cuenta de los cambios alimenticios: alimentación en México, alimentación en Estados Unidos, ocupación y alimentación en Estados Unidos, recursos económicos y alimentación en Estados Unidos.

\section{Resultados}

\section{Alimentación en México}

La alimentación que predomina antes de la migración a Estados Unidos es una dieta basada en los alimentos que se producen en la región en la que viven, tales como: frijol, calabaza, maíz, cacahuate y, en algunas localidades, el pescado. La percepción que tienen las mujeres acerca de esta dieta es que es "comida de pobres" y que además es consumida en poca cantidad por escasez de recursos económicos. Otra percepción es que la comida que ellas consumen en México es fresca, ya que muchos 
de los productos son sembrados y cultivados por su propia familia, o bien obtienen pescado en presas o lagunas presentes en su entorno geográfico. Por su origen campesino, las tortillas que consumen son hechas por las propias mujeres en la vivienda (cuadros II y V).

\section{Alimentación en Estados Unidos}

La alimentación se modifica cuando las mujeres migran a Estados Unidos. Por un lado, conservan alimentos de la cultura mexicana, cuya persistencia es un rasgo de identidad cultural. Sin embargo, la dieta va cambiando en la medida en que se agregan alimentos, sobre todo, que se adquieren con facilidad en los sitios donde viven, o bien que les proporcionan en su lugar de trabajo (hamburguesas, pizzas, sopas instantáneas, comida china) (cuadro II).

\section{Recursos económicos y alimentación en Estados Unidos}

Un elemento clave para el acceso a los alimentos lo constituyen los recursos económicos. Cuando las mujeres se encuentran en Estados Unidos, cuentan con un salario, debido a su inserción laboral, lo que facilita el incremento del abastecimiento de víveres dentro del hogar, así como el consumo de alimentos fuera de la vivienda. El abastecimiento se da a expensas, principalmente, de carnes y lácteos. La obtención de alimentos en gran cantidad es propiciada por el ingreso económico y los enseres domésticos (refrigerador) que permiten almacenarlos. El acceso a recursos económicos, la disponibilidad de grandes supermercados y tiendas de comida rápida en el entorno, juegan un papel importante en el consumo de alimentos (cuadro III).

\section{Ocupación y alimentación en Estados Unidos}

La jornada de trabajo, la intensidad y los horarios de la misma son elementos que influyen para que las mujeres consuman alimentos ya preparados, de los que forman parte de la llamada "comida rápida". Lo anterior se debe a las limitaciones de tiempo para cocinar en el hogar, y se refuerza porque en los mismos sitios en los que laboran (referidos a aquellas mujeres que trabajan en restaurantes), los empleadores les proporcionan alimentos de los que ahí se venden, como un incentivo por desempeñar bien su labor y permitirles, con ello, ahorrar recursos económicos; en ocasiones, esto también les permite trabajar un doble turno. Sin embargo, existen mujeres (sobre todo quienes viven con su pareja o hijos) que cocinan la comida mexicana, y la llevan para comer en el sitio de trabajo (cuadro III).

\section{Traslado social de prácticas alimenticias}

Lo que se encontró fue que las mujeres continúan cocinando platillos mexicanos en Estados Unidos; a algunas de ellas, el consumo de estos alimentos les ayuda para

Cuadro II

Testimonios de alimentación en México y Estados Unidos

\footnotetext{
Alimentación en México

"Extrañaba las tortillas, porque aquí son calientitas y allá son refrigeradas" (Ent 47:I I77-1 I78).

"Allá, pues, comprábamos los paquetes de tortillas y no, aquí es hecha, bueno, yo todavía la hago a mano" (Ent 03:I28-I3I).

“¡Hubo alimentos que comía en México y los quitó cuando se fue a vivir a Estados Unidos?” "Sí: el pescado, la tortilla, el cacahuate, el ajonjolí, que nosotros acá como quiera los comemos. Yo los sembraba, los cultivaba y eso nos hizo falta" (Ent 08:1030-1045).

“Comíamos lo que sembrábamos: frijol, calabaza, maíz, cacahuate, era lo único que hacíamos" (Ent 08:965-969).

"En Chinameca hay un criadero de pescado y abunda mucho, luego era que comíamos pescado en caldo o frito, igual la verdura que hay en el campo como verdolaga o quelites" (Ent 34:I249-I255).

"Comíamos frijoles, salsa, con huevo, con queso; a veces hacíamos pescado; comíamos mucho cacahuate, semillas, chiles secos o chiles frescos y siempre mucha tortilla" (Ent 05:806-816).

Alimentacion en Estados Unidos

"Y cuando se fue a Estados Unidos, ¿cambió sus hábitos de comida?" "Sí, sí cambié mucho por la hamburguesa, pizza, comida china y esos taquitos que les llaman Taco Bell" (Ent 45:322-325).

"Dice que empezó a comer hamburguesas y arroz chino. ¿Usted, antes de ir allá había comido esa comida aquí?" "No, ni los conocía." “¡Algún otro alimento que usted no hubiera comido aquí y empezó a comer allá?" "La sopa Maruchan, el burrito y la pizza” (Ent 40:1029-1046).

"Aquí casi no tomábamos leche, allá casi del diario" (Ent 47:898-900).

"Antes de irse para Estados Unidos, ¿tomaba el refresco?" "Tomábamos, pero muy poco. "¿Y allá?" "Allá sí, todos los días; en la casa y en el restaurante donde trabajaba" (Ent 40:II5I-II64).

"Lo que más cambió en la comida fue que allá comía pizza, hamburguesas; siendo que aquí no lo hay" (Ent 6:528-531).

"Aquí no comía el arroz blanco, el arroz frito. Las pizzas, aquí son otro tipo de masa, allá son diferentes y el pan con mantequilla y ajo. Empecé a comer todo eso y muchos tés fríos y refrescos, tomaba mucho el Doctor Pepers, sabía rico" (Ent 47:I202-1211).

"Además de carne, ¿qué otra cosa empezaron a comprar y a comer que ustedes no comían en México?" "Hamburguesas, pizza y pollo dorado" (Ent 02:|755-|76I)
} 


\section{Cuadro III}

\section{TESTIMONIOS DE RECURSOS ECONÓMICOS Y OCUPACIÓN}

Recursos económicos, accesibilidad y alimentación

"Lo que más comía eran camarones, ostiones, pescado; porque allá hay más modo de comprarlo y donde quiera lo venden, aquí no"(Ent II:1002-1009).

"Luego que las probé me gustaron, y luego el fin de semana le decía a mi esposo: 'Llévame a las hamburguesas', y comía a llenarme, como se dice: a manos llenas" (Ent 32:1160-II64)

"En Estados Unidos es diferente porque uno allá trabaja, tiene dinero, te alcanza para todo. Allá era otra alimentación, pero aquí no, aquí es lo que tengo a la mano" (Ent 8: 427-432).

"Allá teníamos más facilidades de comprar diario leche, fruta y acá no. Aquí el dinero que él gana prácticamente no alcanza" (Ent 04:845-849).

"Pues allá ya hubo más alimento, porque ya compraba mucha fruta para los niños y queso, leche, jugo y carnes. Llenaba el refrigerador de carnes, bisteces, de todo. Entonces había lo suficiente para comer allá" (Ent 29:454-460).

"Allá tiene más facilidad de comprar las cosas porque siempre tiene trabajo. Acá, a veces, nomás lo que hay, porque no hay trabajo y allá va uno comiendo más" (Ent39:259-264).

"Tenía más dinero; por ejemplo, a veces acá nomás una sopa y con tortilla, y allá a veces a la sopa le echaba pollo, papas, zanahoria; acá no, aunque sea nomás la pura sopa y ya, porque a veces pues no alcanza el dinero" (Ent 09:284-291).

"... es difícil porque no hay dinero. Allá si usted ya había comido comida casera, para en la noche decíamos. "¿Saben qué?: coopérense; hay que comprar una pizza o hay que traer hamburguesas'. Entonces aquí ya no... Allá se te facilitaba todo porque solo hablabas por teléfono y ahí venían y todo te traían" (Ent 08:1443-1456)

"Era la misma alimentación porque lo mismo que comprábamos aquí era lo que comprábamos allá. Lo que si aminoró es la cantidad, porque allá trabajando teníamos dinero y aquí estamos más escasos" (Ent 27:1|6|-|167).

Ocupación y alimentación

“QQué comía allá?” "Pues en el trabajo que yo tenía era de comer puras hamburguesas.” “EEn que trabajaba?” "En un McDonald's” (Ent 04:394-404).

"En la fábrica llevaba comida, porque trabajaba con puras negras y luego me hacían burla porque llevaba mi comida y a veces porque me apenaba (por llevar la comida que ella cocinaba) compraba hamburguesa o un pedazo de pizza" (Ent 01:104I-1054).

"A veces al salir del trabajo decía: 'hoy no voy a hacer comida porque estoy cansada', y salía afuera a comer una pizza o hamburguesas." "¡Y cómo qué tan seguido era eso?" "Unas dos o tres veces a la semana" (Ent 29:108I-1096).

"Aquí yo no he comido un plato de camarones. Allá le puedo decir que en cuatro años a lo mejor me comí 50 o 60 platos de camarones y no es que yo los comprara, es que me lo daban en el lugar donde trabajaba como premio por ser buena trabajadora" (Ent 41:1364-1385).

"Yo trabajaba en un restaurante. Cuando yo estaba de descanso, eso era lo que comíamos, la comida china" (Ent 09:392-394).

"La hamburguesa, sí, era diario, con las papas fritas, catsup; de ahí mismo donde trabajaba nos lo daban o, de vez en cuando, cuando descansaba, nos íbamos a comer pizza o comida china" (Ent 06:III6-II22).

“... lo mismo que guisábamos aquí, lo guisábamos allá, que sopa de arroz, papas; lo que nos acomodaba más y llevábamos esa comida al trabajo" (Ent 06:1018-1023).

que su estancia en ese país sea menos difícil. Simultáneamente, con la elaboración de comida mexicana, se van incorporando alimentos propios de la cultura alimenticia de Estados Unidos, especialmente los que son de fácil acceso por su venta y distribución en centros comerciales, así como por la inserción laboral de las mujeres en restaurantes. Sin embargo, se incrementa la cantidad de alimentos que se consumen, tanto del país de origen como del país huésped. Al respecto, muchas mujeres mencionaron que incrementaron su peso, debido al tipo de alimentos que consumieron durante su estancia en Estados Unidos (cuadro V).

Al retornar a México, la mayoría de las mujeres señala que han incorporado a su alimentación algunos platillos que se acostumbraron a comer en Estados Unidos, tales como hamburguesa, pizza, comida china y sopa de fideos precocida. No obstante, los consumen en poca cantidad, debido a la falta de recursos económicos y de sitios en donde comprarlos, aunque algunos migrantes de retorno han abierto sitios para la venta de estos productos, y en las zonas urbanas existen cadenas comerciales que los ofrecen. Algunas mujeres señalaron que sustituyeron el uso de manteca de cerdo por el aceite en la elaboración cotidiana de sus comidas (cuadro IV).

\section{Discusión}

Los resultados de este estudio muestran que los cambios en la alimentación se presentan en ambos sentidos; es decir, durante la estancia de las mujeres en Estados Unidos, acostumbran prácticas alimenticias de la comida mexicana, y cuando las mujeres retornan a México, continúan consumiendo alimentos que fueron incorporados durante su estancia en aquel país, especialmente productos de la llamada "comida rápida". La permanencia de prácticas alimenticias de la cultura mexicana durante su estancia en EUA puede explicarse por tres vías complementarias:

1) La comida está vinculada, como lo señalan diversos autores, ${ }^{16,17,19}$ a la identidad cultural de los grupos sociales.

2) Las comunidades trasnacionales, de las cuales forman parte los migrantes, mantienen contacto estrecho con sus comunidades de origen y con sus 


\section{Cuadro IV \\ Testimonios de traslado social de alimentos México-Estados Unidos-México}

Traslado México-Estados Unidos

"No, mi comida no varió mucho allá, sino que yo allá siempre he comido lo mismo: frijoles, salsa, huevo, tortillas. Hacía tamales. La comida fue la misma, nada más que de vez en cuando comía pizza, hamburguesa o comida china" (Ent 05:734-747).

"Porque si aquí comía tortillas, frijoles, queso, igual allá los comía. Lo que sí, a veces aquí [en México] se come más limitado, se come menos porque no hay trabajo" (Ent 39:25I-256).

"Ya cuando nos daban el break, unos 15 minutos para desayunar algo, comía tortillas con salsa. Ya al mediodía, ya me daban media hora para la comida que nosotros hacíamos, sea caldo de pollo, pollo en arroz, carne de puerco con verduras" (Ent 04:91।-924).

"Allá hacíamos tortillas, pero de Maseca" (Ent 02:1 198).

"La alimentación, yo siento que es lo mismo, porque compra uno sus chiles, papas, jitomates. Allá la diferencia es que se te antoja en la tarde o el fin de semana que una hamburguesa, que una pizza, esa es la diferencia" (Ent 09:76-83).

"Tratamos de hacer moles, comidas, unas picaditas al estilo de México..." (Ent 28: 1466-1469).

"Una vez hice pozole, allá comprábamos enlatado el maíz y luego le poníamos la carne" (Ent 05:449-453).

"Allá luego comíamos carne enchilada, o picaditas para almorzar" (Ent 37:I228-I23I).

"Pues encontrábamos guajes, tunas, la guayaba; aunque nos la daban bien cara porque dicen que les costaba mucho pasar una caja" (Ent 39:236-24I).

"¿Y usted, mientras estuvo en Estados Unidos, qué comía?" "Yo hacía frijoles, arroz, mole, también hacia pipián, mole rojo. Yo compraba el mole de Doña María y le agregaba tomate y chile y salía muy sabroso, seguía preparando mi comida de aquí (México), allá (EUA)” (Ent 33:I305-I326).

"Pues yo compraba los chiles, la carne de puerco, la manteca, el arroz, el chile y hacía lo mismo que comemos aquí, y pues solamente así ya me sentía bien de estar allá, porque al menos ya sabía dónde vendían para hacer comida mexicana" (Ent 16:1021-1028).

\section{Cuadro V \\ CONSUMO Y PERCEPCIÓN DE ALIMENTOS, Y CAMBIO DE PESO CORPORAL EN MUJERES CON EXPERIENCIA MIGRATORIA}

\begin{tabular}{|c|c|c|c|c|c|}
\hline \multicolumn{3}{|c|}{ Alimentación $n=44$} & \multicolumn{3}{|c|}{ Percepción del peso (cambio corporal) $n=44$} \\
\hline México & Estados Unidos & $\begin{array}{c}\text { Percepción sobre la comida de } \\
\text { EE.UU. }\end{array}$ & Antes de EE.UU. & Después de EE.UU. & Causa del cambio de peso \\
\hline $\begin{array}{l}\text { Frutas, verduras, car- } \\
\text { ne de cerdo, queso, } \\
\text { leche, chile, tortilla, } \\
\text { pan, refresco, mante- } \\
\text { ca de cerdo }\end{array}$ & $\begin{array}{l}\text { Carne, pizzas, hamburgue- } \\
\text { sas, espagueti, cereal, comi- } \\
\text { da china, hotdog, hotcakes, } \\
\text { Taco Bell, comida enlatada, } \\
\text { sopa Maruchán, burritos, } \\
\text { arroz chino, pan, pollo ros- } \\
\text { tizado, papas fritas, pollo } \\
\text { Kentucky, golosinas, chetos, } \\
\text { leche, leche de sabor, jugos } \\
\text { embotellados, refrescos }\end{array}$ & $\begin{array}{l}\text { - Allá toda la comida es enlata- } \\
\text { da y aquí es fresca } \\
\text { - Allá la comida está muy vita- } \\
\text { minada, por eso engorda } \\
\text { - La comida tiene muchos } \\
\text { químicos, por eso engorda } \\
\text { - Hay mucha comida chatarra } \\
\text { y las carnes no son frescas } \\
\text { - Alimentación basada en almi- } \\
\text { dones, enlatados y refrigera- } \\
\text { dos, no es saludable } \\
\text { - La carne es desabrida, está } \\
\text { mucho tiempo congelada y } \\
\text { sabe rara }\end{array}$ & $\begin{array}{l}\text { - Gorda } \\
10 / 44=22.7 \% \\
\text { - Delgada } \\
\text { 23/44=52.3\% }\end{array}$ & $\begin{array}{l}\text { - Bajó de peso } \\
4 / 44=9.1 \% \\
\text { - Subió de peso } \\
27 / 44=61.4 \% \\
\text { - Se mantuvo igual } \\
3 / 44=6.8 \%\end{array}$ & $\begin{array}{l}\text { - Por ir a Estados Unidos } \\
\text { (mayor estrés; la cena era } \\
\text { la comida principal; comidas } \\
\text { rápidas; mayor cantidad de } \\
\text { comida) } \\
27 / 44=61.4 \% \\
\text { - Uso de anticonceptivos } \\
\text { |3/44=29.5\% }\end{array}$ \\
\hline
\end{tabular}

compatriotas en el país receptor, formando redes sociales; contribuyen a mantener las comidas y el tipo de alimentación, y refuerzan, así, su sentido de identidad.

2) La tercera vía es lo que P. Bourdieu señala como habitus, el cual se va constituyendo por medio de la experiencia biográfica y conforma un conjunto de esquemas y estructuras mentales que conducen a que se tengan determinadas prácticas; esto, en el caso de la comida, se manifiesta en los gustos y preferencias alimenticias que están vinculadas a la familia y al grupo social al que se pertenece. ${ }^{23}$

Esta permanencia de prácticas alimenticias del país de origen en las mujeres de este estudio es semejante a lo reportado por otras autoras con mujeres migrantes de otras nacionalidades, ${ }^{16}$ lo que refuerza el planteamiento de identidad cultural que posee la alimentación. En contraparte, la incorporación de alimentos tales como pizzas, hamburguesas, pollo frito y comida china, que las mujeres migrantes hacen a su dieta en su estancia en Estados Unidos y que, en menor medida, continúan en México, puede explicarse a su vez, por cuatro vías que se refuerzan entre sí:

1) Esta incorporación puede estar relacionada con la disponibilidad tanto geográfica, como laboral y económica de estos productos. Estos resultados son semejantes a lo reportado por V. Gray y colaboradores, quienes reportaron un incremento en el 
consumo de comidas rápidas debido a la disponibilidad de las mismas y a las rutinas laborales de migrantes mexicanos. ${ }^{9}$

2) Además de la mayor disponibilidad de alimentos en Estados Unidos, se menciona que los mexicanos migrantes toman un modelo de alimentación que fue característico, en otras épocas, de la cultura de este país, y que esta incorporación puede deberse a que los mexicanos suponen que este tipo de alimentación es el mejor en el contexto cultural del país huésped. ${ }^{24}$

3) Diversas autoras han reportado que la inserción laboral de la mujer en Estados Unidos le permite un incremento en la toma de decisiones y mayor autonomía. ${ }^{3,4}$ Las mujeres de esta investigación, debido a los ingresos percibidos, pudieron destinar recursos para consumir alimentos preparados fuera del hogar, lo cual podría ser un indicador de mayor capacidad en la toma de decisiones. En el retorno a México, las mujeres ya no tienen ingresos propios. Sin embargo, debido a que son las responsables de preparar los alimentos para la familia, pueden continuar con prácticas que incorporaron durante su estancia en Estados Unidos.

4) Nuevamente las comunidades trasnacionales parecen jugar un papel en las prácticas alimenticias de las mujeres que retornan a México, ya que en localidades pequeñas, en las que no se encuentran las grandes trasnacionales (McDonalds, Burguer King, Domino's Pizza, entre otras), son los migrantes que retornan quienes venden en establecimientos comerciales propios este tipo de alimentos, y su consumo puede formar parte de una identidad no solo étnica, sino de poblaciones migrantes que los hace diferentes de los propios connacionales que no han tenido experiencia migratoria.

Para el contexto de la venta y distribución de comidas rápidas, B. Popkin señala que, en países de altos ingresos como Estados Unidos, las modificaciones dietéticas -incremento en las porciones alimenticias, consumo de comida fuera de casa, tentempié-están acompañadas de cambios socioeconómicos y demográficos del entorno en general..$^{25}$ Además de estos señalamientos, otro factor es que las cadenas comerciales de comidas rápidas en ese país, a diferencia de Europa, han incrementado la cantidad en las porciones de alimentos y en las bebidas gaseosas que ofrecen al público, ${ }^{26}$ lo cual ha modificado el porcentaje de calorías que se consumen..$^{27}$ Lo anterior tiene gran importancia, debido a que las mujeres migrantes de este estudio consumen con alta frecuencia alimentos en este tipo de establecimientos.
Los cambios en la alimentación de mujeres migrantes tienen consecuencias directas en la salud. Por estudios realizados en Estados Unidos con mujeres migrantes mexicanas, se sabe que las enfermedades cardiovasculares (ECV) son la principal causa de muerte. ${ }^{28}$ En ese sentido, las mujeres mexicanas que residen en Estados Unidos tienen varios de los factores de riesgo para desarrollar ECV tales como: ingesta alta de grasas saturadas, hipertensión y diabetes, ${ }^{29}$ entre otras. Por otro lado, en México, en las últimas décadas, el sobrepeso y la obesidad en las mujeres adultas se han incrementado de manera constante. La Encuesta Nacional de Salud y Nutrición 2006 reportó una prevalencia de $71.9 \%$ al sumar las cifras de sobrepeso y obesidad. ${ }^{30}$ El incremento de este tipo de padecimientos en las mujeres conlleva demandas y necesidades específicas en los servicios de salud tanto en México como en Estados Unidos.

\section{Conclusiones}

Durante su estancia en Estados Unidos, las mujeres mantienen el consumo de alimentos típicos de la cocina mexicana; pero se incrementa el consumo de "comidas rápidas" y, en consecuencia, la ingesta de alimentos densamente energéticos (azúcares concentrados y grasas). Los resultados de este estudio sugieren varios interrogantes y abren otras líneas de investigación: ¿las mujeres migrantes de zonas rurales, acostumbradas al consumo de una dieta escasa y poco variada, durante su estancia en el país de destino, incrementan el consumo de alimentos, como respuesta a las épocas de escasez en el país de origen? ¿Ellas son un grupo blanco para promover el consumo de "comida rápida"? ¿Qué tanto la incorporación de este tipo de alimentos en la dieta de mujeres con experiencia migratoria ha contribuido a los incrementos de sobrepeso y obesidad en México? ¿Qué prácticas alimenticias, tanto del país de origen como de destino, deben fortalecerse en estas mujeres? ¿Qué características deben tener las iniciativas de promoción de la salud y nutrición, dirigidas a este tipo de población tanto en Estados Unidos como en México?

Es necesario profundizar y documentar la relación que existe entre alimentación y migración en el contexto de México-Estados Unidos-México, y proponer iniciativas y políticas públicas que contribuyan a plantear medidas y acciones que mejoren la elección de alimentos de los migrantes en ambos países, antes de la migración y después del retorno.

Declaración de conflicto de intereses. Los autores declararon no tener conflicto de intereses. 


\section{Referencias}

I. Montoya-Zavala E. Migración y desigualdad laboral y salarial por género y estatus migratorio de las sinaloenses en Phoenix, Arizona. Región y sociedad 20I I;23:55-80.

2. International Organization for Migration (IOM). World Migration. Cost and Benefits of International Migration. Geneva: IOM, 2005.

3. Szasz I. La perspectiva de género para el estudio de la migración femenina en México. En: García B (coord.). Mujer, género y población en México. México: El Colegio de México/Somede, 2000:167-204.

4. Solís-Pérez M, Alonso-Meneses G. Una caracterización de las mujeres en tránsito hacia Estados Unidos: 1993-2006. Papeles Poblac 2009;62:253-683. 5. Mellin-Olsen T, Wandel M. Changes in food habits among Pakistani immigrant women in Oslo, Norway. Ethn Health 2005; 10(4):3 I I-339. 6. Pérez-Escamilla R, Putnik P. The Role of Acculturation in Nutrition, Lifestyle, and Incidence of Type 2 Diabetes among Latinos. J Nutr 2007:137:860-870

7. Gil A, Vioque J, Torija E. Usual diet in Bubis, a rural immigrant population of African origin in Madrid. J Hum Nutr Diet 2005; |8(I):25-32. 8. Day K. Active Living and Social Justice Planning for Physical Activity in Low-income,

Black, and Latino Communities. J Am Plann Assoc 2006;72(I):88-99. 9. Gray VB, Cossman JS, Dodson WL, Byrd SH. Dietary Acculturation of Hispanic Immigrants in Mississippi. Salud Publica Mex 2005;47:;35I-360.

10. John EM, Phipps Al, Davis A, Koo J. Migration History, Acculturation, and Breast Cancer

Risk in Hispanic Women. Cancer Epidemiol Biomarkers Prev 2005; I 4(I2):2905-29|3.

II. Singh GK, Miller BA. Health, Life Expectancy, and Mortality Patterns Among Immigrant Populations in the United States. Can J Public Health 2004;95(3): I- I5

12. Yang EJ, Kerver JM, Song WO. Dietary Patterns of Korean Americans Described by Factor Analysis. J Am Coll Nutr 2005;24(2):I I5-I2I. 13. Kiyah DJ, Gordon-Larsen P, Ayala GX, Popkin BM. Birthplace is Associated with More Adverse Dietary Profiles for US-Born than for Foreign-Born Latino Adults. J. Nutric 2008; 138:2428-2435.

14. Mennell S, Murcott A, Van Otterloo AH. The Impact on Food of Colonialism and Migration. The Sociology of Food: Eating, Diet and Culture. Curr Sociol 1992;40(2):75-80.

15. Levenstein Harvey A. The American Response to Italian Food, I8801930. Food Foodways 1985; I(I): I-24
16. Vallianatos H, Raine K. Consuming food and Constructing Identities Among Arabic and South Asian Immigrant Women. Food, Cult Society 2008; II (3):355-373.

17. Calvo M. Migration et alimentation. Soc Sci Inf (Paris) 1982;21(3):383-446

18. Ariza M. Migración, familia y trasnacionalidad en el contexto de la globalización: algunos puntos de reflexión. Rev Mex Sociol 2002;64(4):53-84. 19. Giorgulli Saucedo S E, Itzigsohn J. Diferencias de género en la experiencia migratoria. Trasnacionalismo e incorporación de los migrantes latinos en Estados Unidos. Papeles Poblac 2006;47:9-37.

20. Bertran Vila M. Migración y hábitos alimenticios en la ciudad de México: un acercamiento antropológico. En: Romero Martínez A, Torre Medina-Mora MP, Muñoz-Vega VM, Espíndola-Frausto D, Comp. Quehacer científico. Un panorrama actual en la UAM-X Tomo II. México, DF: UAM-X, 2004:173-|8|.

2I. Reyes Posadas I, Nazar Beutelspcher A, Estrada Lugo E, Mundo Rosas V. Alimentación y suficiencia energética en indígenas migrantes de los Altos de Chiapas, México. Archivos Latinoamericanos de Nutrición. 22. Strauss A, Corbin J. Basics of qualitative research: Techniques and procedures for developing grounded theory. 2a. edición. London: Sage Publication, 1999.

23. Bourdieu P. Cuestiones de sociología. Madrid: Itsmo, 2000.

24. Wallendor FM, Reilly MD. Ethnic migration, assimilation, and consumption. Journal of Consumer Research 1993;10:292-302.

25. Popkin Barry M. Global nutrition dynamics: the world is shifting rapidly toward a diet linked with noncommunicable diseases. Am J Clin Nutr 2006;84:299-298.

26. Young LR, Nestle M. Portion Sizes and Obesity: Responses of FastFood Companies. J Public Health Policy 2007;28:238-248.

27. Kiyah J. Duffey, Barry M Popkin. Shifts in Patterns and Consumption of Beverages Between 1965 and 2002. Obesity 2007;15:2739-2747.

28. Juarbe TC. Cardiovascular Disease Related Diet and Exercise Experiences of Immigrant Mexican Women. West J Nurs Res 1998;20(6)765-82 29. Pérez-Stable EJ, Juarbe TC, Moreno-John G. Cardiovascular Disease. En: Molina MA (ed.). Health Issues in the Latino Community. San Francisco: Jossey-Bass, 200I:245-276

30. Olaiz-Fernández G, Rivera-Dommarco J, Shamah-Levy T, Rojas R, Villalpando-Hernández S, Hernández-Avila M, et al. Encuesta Nacional de Salud y Nutrición 2006. Cuernavaca: Instituto Nacional de Salud Pública, 2006. 